\title{
Observations of the Sun's Dragging Forces
}

\author{
Jose L. Parra \\ Physics Department, Florida International University, Miami, USA \\ Email: JLparra@fiu.edu
}

How to cite this paper: Parra, J.L. (2020) Observations of the Sun's Dragging Forces. Journal of Applied Mathematics and Physics, 8, 643-651.

https://doi.org/10.4236/jamp.2020.84049

Received: March 4, 2020

Accepted: March 29, 2020

Published: April 2, 2020

Copyright (C) 2020 by author(s) and Scientific Research Publishing Inc. This work is licensed under the Creative Commons Attribution International License (CC BY 4.0).

http://creativecommons.org/licenses/by/4.0/

\begin{abstract}
The classical theory of gravity, developed by Isaac Newton, predicts that the gravitational force between two masses is always colinear with the direction defined by the center of mass of both bodies. Some work done in the last 60 years has shown experimental evidence that the reality may be somehow more complex. That complexity was confirmed by the author of this paper and he goes farther announcing that the Sun is emitting particles with $44 \mathrm{~m}$ spatial periodicity that creates isotropic gravitational effects. Those effects are identical to the ones produced by dragging forces according to the General Theory of Relativity under the Kerr's Metric. The purpose of this paper is to introduce experimental evidence confirming the author's predictions that the Moon can modify the dragging force coming from the Sun's core.
\end{abstract}

\section{Keywords \\ Gravity, Universal Gravitational Constant, Diffraction, Interference, Dragging Forces}

\section{Introduction}

The survival of the human being is strongly dependent on the Earth crossing paths with meteorites, comets, and the Sun's flares. This is globally accepted, and some governments give serious attention to those events. However, it is not enough. Unfortunately, the fusion process inside the Sun, our source of light and heat, by various orders of magnitude is more dangerous than the previously mentioned threats. Any minuscule variation on the fusion rate, according to the Sun standard, will be seen here on Earth as a dramatic temperature variation that could endanger the future of life as we know it. Neutrino counting is a very expensive technique, but it is the only way used today to characterize the fusion rate occurring in the Sun. In the author's opinion, any report about what is happening in the Sun, even the smallest variation of the Sun's activity, should be considered important because it could open another window to our under- 
standing of our source of life (or death). This paper is about the history of one of those outcomes coming from the Sun and its physical characterization, which is communicated for the first time here.

This idea about an unknown energy coming from the Sun is not new in physics. Some peers around the world have detected some experimental "anomalies" that cannot be explained by using the classical physics. Here are introduced some of those reported anomalies. In 1959 Alois [1], a Nobel Prize winner, announced the observation of anomalies in the motion of a paraconical pendulum during a Total Solar Eclipse (TSE), and became the first to announce, "the existence of a new field." Jeverdan et al. [2] published a variation of the period of a Foucault's pendulum on the fifth significant figure, where there is a visible minimum during the maximum occultation of the Sun. Saxl and Allen [3], using a torsion pendulum, reported variation on the fourth significant figure of its period during the 1971 TSE. Yang and Wang [4] mentioned vertical gravity variations during the 1997 TSE with excellent experimental clarity. Also, Duval [5] mentioned similar vertical effects during the 1994 TSE. Goodey et al. [6] presented abundant experimental evidence of change of behavior of different instruments widely separated; the 2008 TSE observed by them, showed remarkable synchronized reactions between the different settings. Pugach and Olenici [7] completely confirmed Goodey et al.'s results with stronger time, shape, and lapse correlations on the 2009 TSE that occurred far away from the instruments used. Heck et al. [8] used a paraconical pendulum from 2005 to 2007, covering a TSE on March $3^{\text {rd }}, 2007$ and other astronomical combinations. They did not observe any significant variation away from classical physics. Heck et al. concluded that all the gravitational anomalies should be produced by "erratic disturbances and vibrations in the surroundings" of the paraconical pendulum.

All studies about the anomalous gravitational signals, references [1]-[8] and others, bias the author to three conclusions:

1) Authors [1]-[7] report weak signals and [8] no signal at all, indicating that the experimental tools used were not the most appropriate ones to study those gravitational phenomena.

2) The observation period should be large, as done by [8], but with a frequency of observation close to the frequency of variation of the signal.

3) Authors [1]-[7] fail to report a model and the values of its physical parameters. Without any model it is hard to make predictions.

The author tried to turnover from these three conclusions. He, by trial and error, designed a reliable equipment that output abundant and clear data about the gravitational perturbations created by the Sun on the Earth's surface. The idea was to create a mechanical filter that could clearly display the signal of interest and quickly neutralize the undesired ones. That was achieved by four modifications of a Cavendish-type balance. Two metallic filaments, instead of one, were used to maintain the balance in horizontal equilibrium. The separation between the two filaments, again by trial and error, was changed until the filter was ready. The second modification was the use of an electronic micro- 
scope connected to a computer to do digital recording. The third one was the use of cylindrical disks with flat planes in the vertical direction; in that way the gravitational attractions between the masses were increased and the amplitude of the oscillations decreased because a stopping force appeared on the circular superficies of the balancing masses acting as sails. The fourth one was to keep the position of the attractor masses fixed to the base to reduce the experimental errors on position. This idea made very hard to measure Big G, which, anyway, the author did not intend to do. However, this technique made clear that any variation on the position of the pendulum masses must be produced by external forces. This setting improved the data collection process by two orders of magnitude with respect to the best previous work on gravitational anomalies.

A central point on the oscillating cylinder was selected to be the zero point on the first day of observation. After that, the position in pixels of that point was recorded every day. Figure 1 included more than 1000 positions recorded from December 2016 to October 2019. This Figure 1 by itself indicates that the experimental data is reliable, and that an anomalous gravitational force is decreasing with the distance to the Sun and changing with the cosine of an angle that symmetrically goes around 90 degrees. The peaks of Figure 1 show some correlation with preferred position of the Moon. The positions of the Moon on those preferred points indicate that the dragging force from the Sun experiences reflection and diffraction phenomena. Details about the mentioned correlation will be covered on another paper.

The author will assume that a plasmatic torus is rotating at relativistic speed at

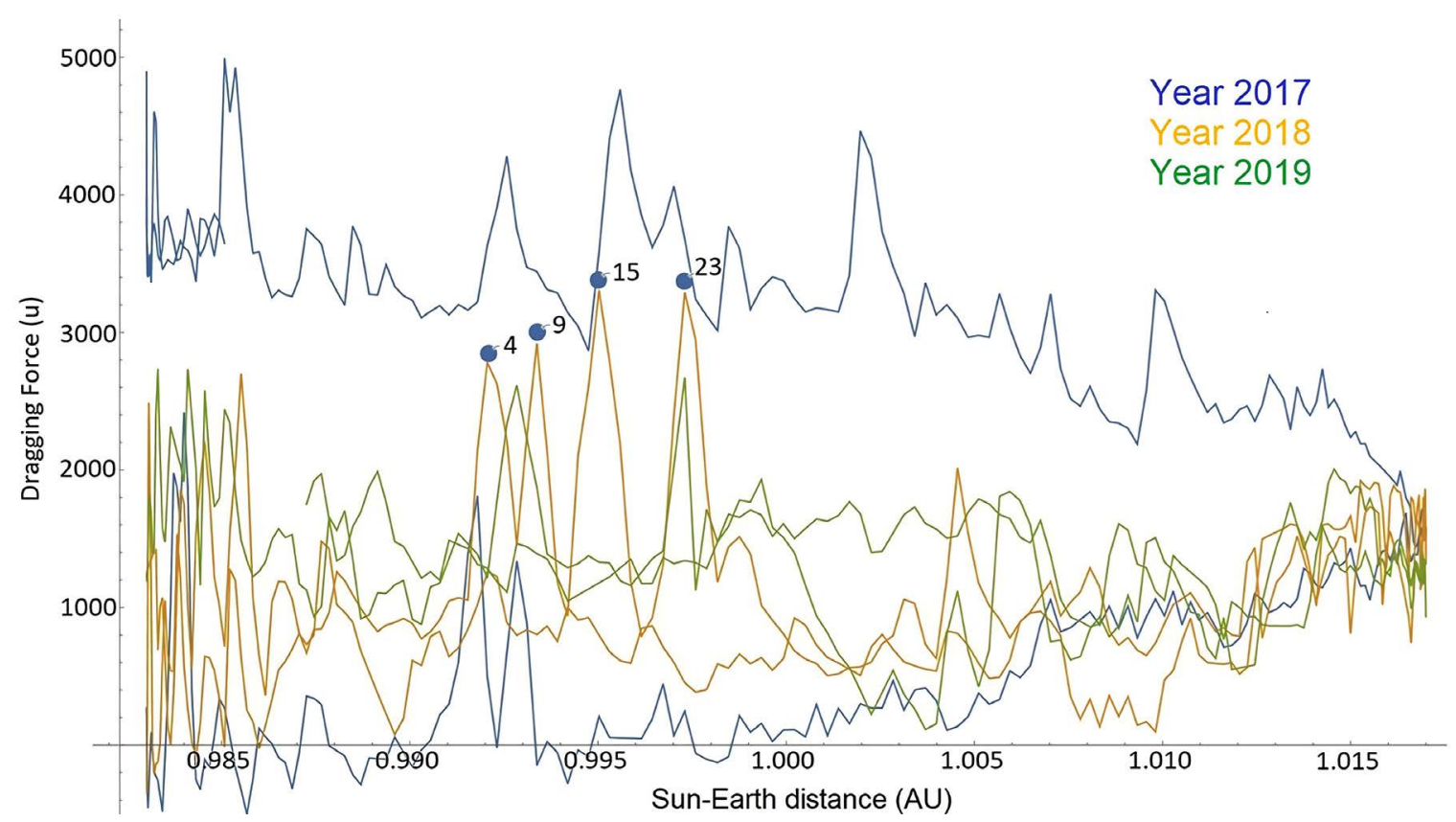

Figure 1. Dragging force on one of a selected point on one of the masses of a Cavendish-type balance versus the distance to the Sun. All blue spots represent dates in the year 2018. Spot 4 on March $4^{\text {th }}$, spot 9 on March $9^{\text {th }}$, spot 15 on March $15^{\text {th }}$, and spot 23 on March $23^{\text {rd }}$. The height of those yellow peaks indicated that something huge was happening in the nucleus of the Sun. 
the center of the Sun. Kerr [9] found the solution of this situation by using the General Theory of Relativity of Albert Einstein [10]. His solution predicts a dragging torque on bodies with extension, or dragging forces acting on moving point-masses. The dragging idea was introduced by the author in [11] and clarified with Equation (3) of [12]. Wu et al. [13] wrote a review paper mentioning the possibility that the determination of the Universal Gravitational Constant, also called Big G, could be affected by this dragging force. More importantly, they clarify that more data is necessary in order to support this point of view. The following topic is a modest fulfillment of their request for additional data.

\section{Eclipses}

\subsection{Observations}

The author, based on the reliability of their daily experimental observations, was inspired to study the screening effect of the Moon on the dragging force coming from the Sun. On August 21 $1^{\text {st }}, 2017$ a TSE occurred in the USA. That day, observations of the dragging force were done every 15 minutes, from 5:00 A.M. to 8:00 P.M.

A clear pattern with maxima and minima becomes visible. Those maxima and minima could be equivalent to the positive and negative values reported in [1]-[7] at their corresponding Total Solar Eclipse (TSE) time events.

The author, to the best of his knowledge, claim that this pattern can only be explained by a combination of photonic gravitational diffraction on the rim of the Moon and photonic interference on the Moon post-space. The other possible explanation assuming gravitational space-time waves is not a good candidate because the Sun is not massive enough to create waves with intensity close to the signal detected.

The matching between the experimental data and the theoretical model motivated the author to predict that more information will be noticed during solar eclipses if the signal is recorded thru bigger periods of time. On July $2^{\text {nd }}, 2019$, a new opportunity appeared over Argentina. The author made a second version of the modified Cavendish balance and moved it to the hotel Patric, in the city of Chivilcoy. The original set stayed working automatically at Florida International University (FIU). The computer on Chivilcoy recorded a picture every two seconds and the one in FIU every ten seconds because both settings had different restoring constants. The results were astonishing as can be recognized by observing Figure 2.

The synchronism between rounded peaks, valleys, and central large narrow peaks (with blue and yellow colors) of both curves is big and excluded any casual coincidence. The direct conclusion from this observation is that a real signal of cosmological proportion is coming from the Sun and, because the central role played by the Sun onto life on Earth, its variations should be recorded globally.

\subsection{Parametrization of the Dragging Force}

The author in this paper hypothesized that the corpuscular interaction between 


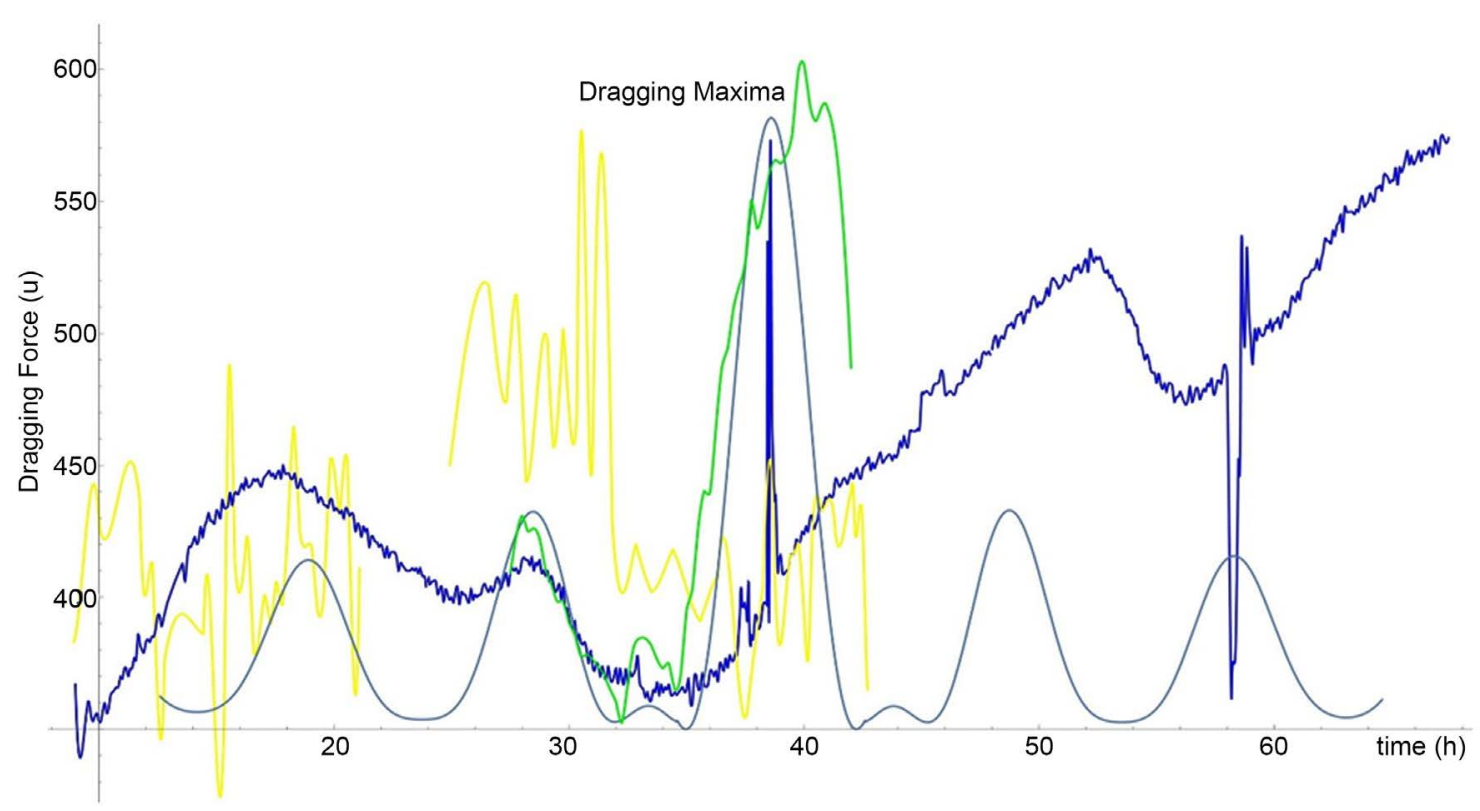

Figure 2. Green is the data from the Cavendish type balance at FIU for the TSE on August $21^{\text {st }}, 2017$. The dark-blue line is the data from same balance at FIU for the TSE on July $2^{\text {nd }}, 2019$. The yellow line is the data from the Cavendish type balance at Chivilcoy (Argentina) for the TSE, on July $2^{\text {nd }}, 2019$, and the light-blue curve from Equation (1) and Equation (2).

approaching photons are the only bias to the photon's redistribution; see [14] for details. Let's assume that photons are coming from the Sun in phase. The ones moving inside a circular deflecting region of height $h$ above the surface of the Moon could experience reflection, with a variation of its phase, or could experience refraction, without change in their phase. Photons moving to points below the height $h$ will merge with other photons; all of them suffered refraction. In any point above $h$ will merge photons coming strike from the Sun with others that suffered reflection from the closer side of the moon and others that suffer refraction from the farther side of the Moon. The total amplitude in any point was calculated by integrating all possible trajectories; the amplitude square became the intensity of the signal in that point. See Equation (1) for the intensity of points on the shadow region and Equation (2) for the intensity of points on the illuminate region.

$$
\begin{aligned}
& \left.I_{S}\right|_{r \leq R_{M}+h_{\text {def }}} \approx A^{2}\left\{\left[\int_{0}^{2 \pi} \frac{x}{l} \cos \left(\frac{2 \pi l}{\lambda}\right) \mathrm{d} \theta\right]^{2}+\left[\int_{0}^{2 \pi} \frac{x}{l} \sin \left(\frac{2 \pi l}{\lambda}\right) \mathrm{d} \theta\right]^{2}\right\}
\end{aligned}
$$

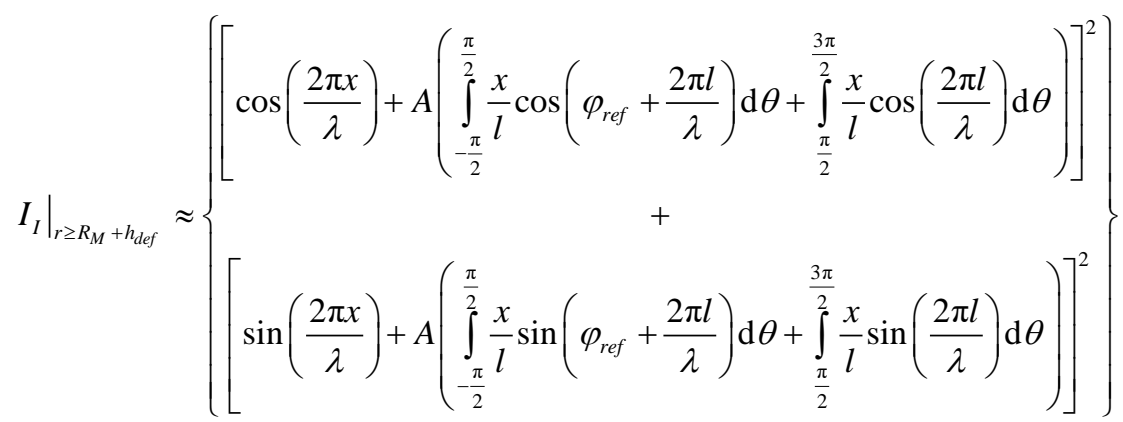


where $A$ is the amplitude of the incoming signal, $x$ is the Moon-Earth distance, $I$ is the $3 \mathrm{D}$ distance between two points, namely

$$
l=\sqrt{x^{2}+\left[\left(R_{M}+h_{d e f}\right) \cos (\theta)-r\right]^{2}+\left[\left(R_{M}+h_{d e f}\right) \sin (\theta)\right]^{2}}
$$

$R_{M}$ is the radius of the Moon, $h_{d e f}$ is the height of the region where deflection, by reflection or refraction, happens, $r$ is the radial distance to the axis passing by the center of the Moon, $\lambda$ is the spatial period of the dragging force coming from the Sun, $\varphi_{\text {ref }}$ is the extra phase added only after reflection, and $\theta$ is the angle of integration around the rim of the Moon. See Figure 3 for details.

Figure 4 was done using the software Mathematica and it included intensities

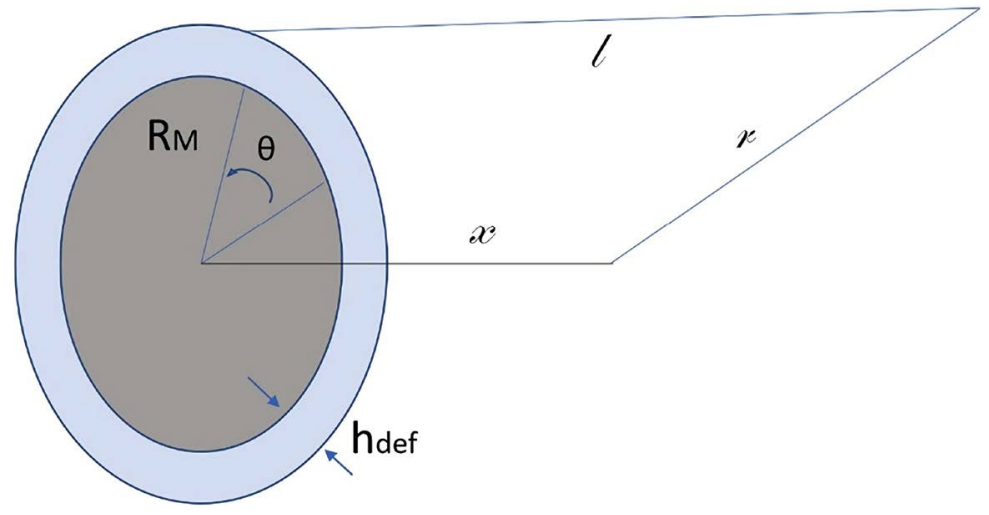

Figure 3. Necessary parameters to calculate the intensity of the dragging forces at a distance $r$ from the center of a celestial body as the Moon or the Earth. Earth is mentioned here because its dragging shadow will be reflected later by the Moon and come back to the Earth.

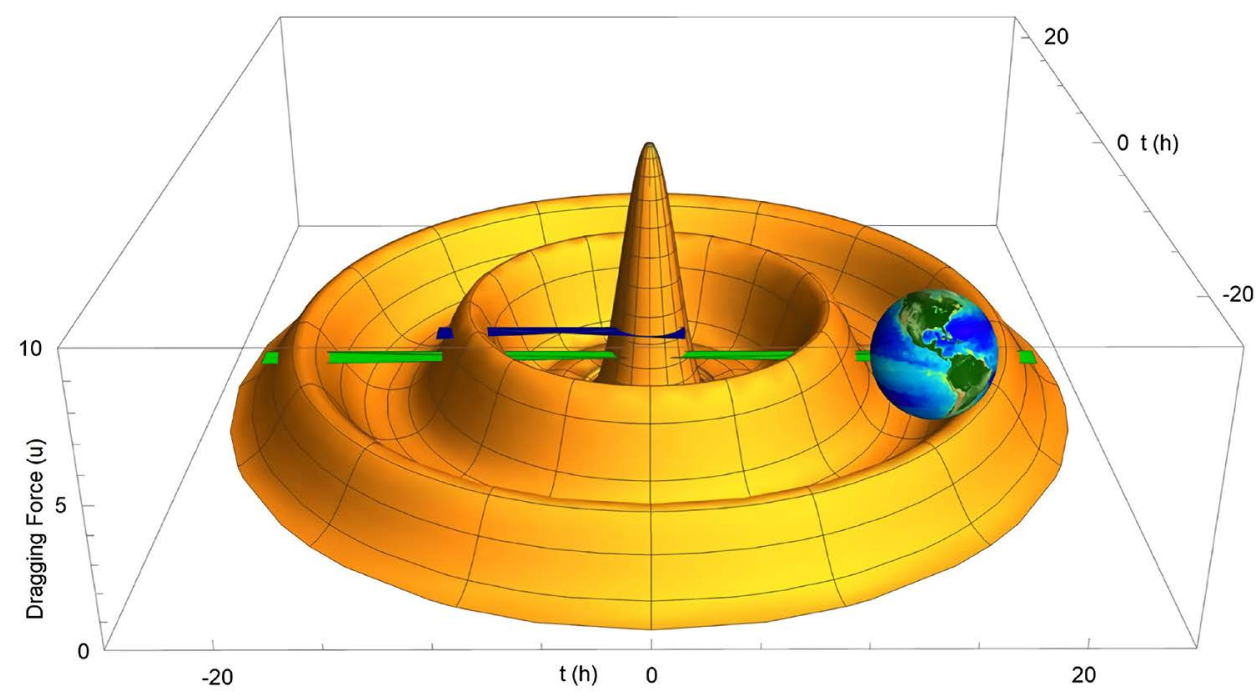

Figure 4. Interference pattern of the dragging force overpassing the Moon, as shown here, is always happening independently if we are having a TSE on Earth or not. It is, most of the time we do not detect amplify dragging forces because the path of the optical umbra of the Moon does not cover the surface of the Earth. Blue line for the August $21^{\text {st }}, 2017$ TSE path and green line is for the one on July $2^{\text {nd }}, 2019$. 
according to Equation (1) and Equation (2) and the path followed by Earth on the TSE of 2017 and 2019. The image of the Earth [15] was added to Figure 4 to compare sizes. The values of the parameters used were $A=\pi / 100 \mathrm{u}, R_{M}=1750$ $\mathrm{km}, h_{\text {def }}=4 \mathrm{~km}, \lambda=44 \mathrm{~m}$, and $\varphi_{\text {def }}=-0.5 \mathrm{rad}$. The value of the partial amplitude $A$ was defined by dividing every semicircle in 100 parts; in that way, the total amplitude was normalized to one. The deflection phase was set as -0.5 to satisfice the continuity of the signals under the shadowed region of the Moon and the illuminated region outside of the Moon. Finally, the value of the spatial period $\lambda$ was found by try and error; the main criteria were to overlap maxima and minima of the model with its experimental corresponding. The transformation for space-time scales was defined by the average velocity of the shadow of the Moon, it is $2250 \mathrm{~km} \cdot \mathrm{h}^{-1}$. To clarify, the center of the Moon will cover the $12,756 \mathrm{~km}$ diameter of the Earth in $5.67 \mathrm{~h}$. At this time, it will be convenient to mention that the optical shadow of the Moon is not coincident in space with the interference pattern produced by the dragging force; this observation was mentioned before by Pugach [7].

\subsection{Eclipses and Model}

The relevance of this paper can be perceived by combining in a graph all the experimental data and the mathematical model developed to describe the nature of the dragging force. Something peculiar about Figure 2 is the bigger size of the dragging signals after the dragging maximum. This can be justified by the spherical shape of Earth and the variation of the relative velocities between the Earth and the Moon.

Any Total Solar Eclipse is by nature a singular astronomical event connecting three massive bodies in a specific way. Those bodies are the Sun as a source, the Moon as a possible perturbator, and the Earth as a receiver. The experimental observation of gravitational horizontal torques during two TSE made by three independent equipment totally confirm the existence of a dragging force coming from the Sun and modified by the Moon. The existence of maxima and minima pointed out the nature of the mention signal because it means the observation of diffraction and interference phenomena. The explanation of that pattern could be done by using a wave or a particle point of view. Correlation of the mass of the Sun with the intensity of the signal detected here on Earth could be a good discriminator. It supported the idea of the dragging energy traveling in packs and invalidated the assumption of that energy dispersing in space as a wave.

\section{Conclusions}

It has now been 60 years since the first announcement that an anomalous gravitational effect has been detected by Allais [1]. After that, many other peers documented its existence in different countries and claimed simultaneous observations. In 2016, the author of this paper decided to do some gravitational experiments in relation to the lack of consensus about the value of the universal con- 
stant Big G. Because the author had no information about the strange gravitational anomaly reported before, he made his own equipment in a different way. By coincidence that equipment became very sensitive to variations of any horizontal force, gravitational or not. Another advantage, as intended by the designer, was the linear reaction of the equipment to forces changing on large periods of time, coupling to its filtering effect of any short time variable force.

A pattern of the studied signal became clear with the accumulation of data. The author claims that the signals detected are not anomalous at all but closely follow the dragging force predicted by the Kerr's Metric. After the experimental check that the dragging force changes with the distance to the Sun, the authors observed that the signal peaks show some correlation with the position of the Moon. The next logical step was to study what happens to the dragging force when the Moon eclipses the Sun. The outcome of a pattern of interference around the time of the Total Solar Eclipse (TSE) of 2017 in the United States makes the connection between all the "anomalous" gravitational effects previously detected. It is the positive effects that can be correlated with constructive interference and the negative effects of destructive interference.

The total confirmation of the author's prediction that longer time of observation during the 2019 TSE would produce the same pattern but with more information bolstered the author's hypothesis. Two TSEs and three locations of observation in complete agreement make reliable the conclusion that the Sun is emitting particles with potential gravitational energy having $44 \mathrm{~m}$ of spatial periodicity.

\section{Acknowledgements}

My sincerest gratitude to the Center for Excellence in Writing, Florida International University, for their careful dedication during the edition of this paper. Special recognition to college F. Muller for detailed discussion of the ideas introduced in this paper.

\section{Conflicts of Interest}

The author declares no conflicts of interest regarding the publication of this paper.

\section{References}

[1] Allais, M. (1959) Should the Laws of Gravitation Be Reconsidered? Part II Abnormalities in the Motion of a Paraconical Pendulum on an Anisotropic Support. Aero/ Space Engineering, 46, 51-56.

[2] Jeverdan, G.T., Rusu, G.I. and Antonescu, V. (1961) Experiments Using the Foucault Pendulum during the Solar Eclipse of 15 February, 1961. The Biblical Astronomer, 1, 18-20.

[3] Saxl, E.J. and Allen, M. (1971) 1970 Solar Eclipse as "Seen" by a Torsion Pendulum. Physical Review D, 3, 823-825. https://doi.org/10.1103/PhysRevD.3.823

[4] Yang, X. and Wang, Q. (2002) Gravity Anomaly during the Mohe Total Solar Ec- 
lipse and New Constraint on Gravitational Shielding Parameter. Astrophysics and Space Science, 282, 245-253. https://doi.org/10.1023/A:1021119023985

[5] Duval, M. (2005) An Experimental Gravimetric Result for the Revival of Corpuscular Theory. Physics Essays, 18, 53-62. https://doi.org/10.4006/1.3025724

[6] Goodey, T.J., Pugach, A.F. and Olenici, D. (2010) Correlated Anomalous Effects Observed during the August 1st 2008 Solar Eclipse. Journal of Advanced Research in Physics, 1, 1-7.

[7] Pugach, A.F. and Olenici, D. (2012) Observations of Correlated Behavior of Two Light Torsion Balances and a Paraconical Pendulum in Separate Locations during the Solar Eclipse of January 26th, 2009. Advances in Astronomy, 2012, Article ID: 263818. https://doi.org/10.1155/2012/263818

[8] Heck, A. (2009) The Paraconical Pendulum (Allais-Effect) Reconsidered. Gravitation.org. 1-8.

[9] Kerr, R.P. (1963) Gravitational Field of a Spinning Mass as an Example of Algebraically Special Metrics. Physical Review Letters, 11, 237-238.

https://doi.org/10.1103/PhysRevLett.11.237

[10] Einstein, A. (1916) Die Grundlage der allgemeinen Relativitätstheorie. Annalen der Physik, 354, 81-124. https://doi.org/10.1002/andp.19163540702

[11] Parra, J.L. (2017) The Implications of the Sun's Dragging Effect on Gravitational Experiments. International Journal of Astronomy and Astrophysics, 7, 174-184. https://doi.org/10.4236/ijaa.2017.73014

[12] Parra, J.L. (2018) Toward a Common Ground for Gravity and Optics. Journal of Applied Mathematics and Physics, 6, 1896-1906.

https://doi.org/10.4236/jamp.2018.69161

[13] Wu, J., Li, Q., Liu, J., Xue, C., Yang, S., Shao, C., Tu, L., Hu, Z. and Luo, J. (2019) Progress in Precise Measurements of the Gravitational Constant. Annalen der Physik, 531, Article ID: 1900013. https://doi.org/10.1002/andp.201900013

[14] Parra, J.L. (2018) Corpuscular Point of View to Explain Light's Properties. Optics and Photonics Journal, 8, 135-145. https://doi.org/10.4236/opj.2018.85013

[15] NASA (2017) Earth: Our Living Planet. NASA Solar System Exploration. https://solarsystem.nasa.gov/resources/373/earth-our-living-planet 